\title{
Association Between Micronutrient Concentrations and Human Epididymis Protein 4
}

\author{
Zhu Wei Lim' \\ Wei Liang Chen (iD ${ }^{2}$ \\ 'Department of Obstetrics and \\ Gynecology, Changhua Christian \\ Hospital, Changhua, Taiwan; ${ }^{2}$ Division of \\ Geriatric Medicine, Department of Family \\ and Community Medicine, Tri-Service \\ General Hospital and School of Medicine, \\ National Defense Medical Center, Taipei, \\ Taiwan
}

Background: Human epididymis protein 4 (HE4) has been frequently used to study in many malignant tumors, while serum nutritional markers are used to determine a person's health status. However, the link between serum micronutrient concentrations and HE4 has not yet been clarified.

Methods: A total of 2464 eligible female participants and serum concentrations of nutritional biomarkers were chosen from the National Health and Nutrition Examination Surveys (NHANES) 2001-2002. For statistical analysis, we used the $\chi^{2}$ test, multivariable linear regression, and analysis of variance. Adjusted models were used, and the concentrations of serum nutritional biomarkers were divided into quartiles.

Results: The mean age of the participants was 48.07 years. Among twelve micronutrients, five were negatively associated with HE4 in models 1,2 and 3. Only $\alpha$-carotene, trans- $\beta$ carotene, cis- $\beta$-carotene, trans-lycopene and retinol were associated with HE4, with beta coefficients of $-0.102,-0.027,-0.506,-0.131$ and -0.054 , respectively. After performing quartile-based analysis, statistical significance was only found for serum $\alpha$-carotene, translycopene, and retinol in the three models. In model 3, the beta coefficients [95\% confidence intervals (CIs)] of the fourth quartiles compared to the first quartiles for $\alpha$-carotene, translycopene, and retinol were $-3.390(-5.053,-1.727),-4.036(-5.722,-2.351)$ and -4.146 $(-5.899,-2.393)$, respectively. Serum concentrations of these three nutritional biomarkers were inversely related to serum HE4 concentration ( $p$ trend $<0.001$ ).

Conclusion: HE4 is a useful and novel biomarker that can be used with many diseases, especially ovarian cancer. Three of our selected micronutrients were inversely associated with HE4 concentration. Supplement of micronutrients may reduce the levels of HE4 and the subsequent of ovarian cancer's risk. Therefore, a formula that correlates HE4 with nutritional biomarkers needs to be established before use in clinical applications.

Keywords: human epididymis protein 4, vitamin A, vitamin E, carotenoids

\section{Introduction}

CA 125, HE4, apoptosis repeat baculoviral inhibitor-5, e-cadherin, mucin 1 and prostasin were a list of important biomarkers for ovarian cancer. ${ }^{1}$ Due to multiple biomarkers were used in the detection of ovarian cancer, nanomaterial-based biosensors were developed to identifying and controlling them. ${ }^{1}$ They can convert chemical analyte into signal, detect limited range of biomarkers to have a more beneficial result. ${ }^{1}$ Human Epididymis Protein 4 (HE4) has been developed as a biomarker to detect ovarian cancer because the positive predictive value of cancer antigen 125 (CA125) for ovarian cancer is only $10.1 \%{ }^{2}$ CA125 may also increase in benign gynecological disease, menstruation, pregnancy, heart failure and liver
Correspondence: Wei Liang Chen Division of Geriatric Medicine, Department of Family and Community Medicine, Tri-Service General Hospital and School of Medicine, National Defense Medical Center, Taipei, Taiwan

Tel +886-2-879233II ext. 16567

$\mathrm{Fax}+886-2-87927057$

Email weiliang0508@gmail.com 
cirrhosis. ${ }^{3}$ HE4 expression is detected less often in benign diseases of the uterine corpus, breast cancer, gastrointestinal tumors and urinary tract tumors. ${ }^{4}$

Nutrient-rich supplements such as carotenoids and vitamins are used commercially because of their medicinal properties and the increasing health consciousness of the population. Carotenoids have potent cytotoxic effects against various cancer cells. Their associated mechanisms include enhanced oxidative stress and apoptosis, effects on the MAPK/ERK pathway, the overexpression of proapoptotic proteins, the overexpression of tumor suppression proteins, the suppression of metastasis and cell cycle arrest. ${ }^{5}$ In addition, carotenoids, as serum nutritional biomarkers, have consistent associations with sleep quality and quantity. ${ }^{6}$

HE4 and carotenoids share similar signaling pathways. The association between carotenoids and HE4 has been underinvestigated. The possible associated pathways and mechanisms including cell proliferation, tumor growth, metastatic invasion, migration, adhesion, and chemoresistance. ${ }^{7}$ Therefore, our aim in this study was to examine the link between carotenoid levels and HE4 concentration.

\section{Methods}

\section{Design and Participants}

The National Health and Nutrition Examination Survey (NHANES) is an ongoing cross-sectional survey of the United States population. ${ }^{8}$ A total of 11,039 participants of all ages were included in the NHANES 2001-2002 database. Data collection, physical examination and laboratory investigation were completed after the participants had provided their consent personally. All NHANES participants underwent two-part household interviews. The first part proceeded with a trained interviewer, while the second part was conducted with a computer or other technology. The information included age, gender, race, medical history, health status, smoking, drug use, alcohol, and sexual behavior. Further physical examinations and laboratory tests were performed at a mobile examination center (MEC). For more detailed information, please visit the NHANES 2001-2002 website, https://wwwn.cdc.gov/nchs/nhanes/ continuousnhanes/default.aspx?BeginYear=2001. The NHANES data were approved by the Institutional Review Board (IRB) of the National Center for Health Statistics (NCHS).

\section{Measurement of Nutritional Biomarkers}

Serum nutritional biomarkers were processed, stored at $20{ }^{\circ} \mathrm{C}$, and shipped for analysis and testing according to the NHANES' protocol. A total of twelve micronutrients, including retinol, $\alpha$-tocopherol, $\gamma$-tocopherol, retinyl palmitate, retinyl stearate, $\alpha$-carotene, trans- $\beta$-carotene, cis $\beta$-carotene, $\beta$-cryptoxanthin, lutein/zeaxanthin, and translycopene, were examined according to the NHANES' laboratory methodology.

The NHANES has collected data on serum nutritional biomarkers since 1971. Serum retinol was uncommon in individuals aged 4 years and older, but variations in racial/ ethnic and socioeconomic differences were found in NHANES III (1988-1994), ${ }^{9}$ as well as variations in serum carotenoid concentrations. ${ }^{10}$

The common cutoff values for serum vitamin A, vitamin $\mathrm{E}$ and serum $\alpha$-tocopherol concentrations were $20 \mu \mathrm{g} \mathrm{dl}^{-1}, 500 \mu \mathrm{g} \mathrm{dl}^{-1}$ and $500 \mu \mathrm{g} / \mathrm{dL}$, respectively. ${ }^{11}$

Other fat-soluble micronutrients had different reference values between children, adolescents, adults and older people ( $\geq 60$ years). ${ }^{11}$ In general, vitamin $\mathrm{A}$ and $\mathrm{E}$ were lower in children and adolescents; $\alpha$-carotene and lutein/ zeaxanthin were lower in adolescents, $\beta$-carotene was lower in adolescents and in adults 20-39 years old, translycopene and $\gamma$-tocopherol were lower in young children and older people, and $\beta$-cryptoxanthin was lower in adolescents or adults. ${ }^{12}$

The interpretation of various serum nutritional biomarkers between races was as follows: vitamin $\mathrm{A}$ and $\gamma$-tocopherol were lower in Mexican Americans and non-Hispanic whites, while vitamin E was lower in non-Hispanic blacks. ${ }^{12}$ The assay methodology and reference values for the nutritional biomarkers are discussed in more detail on the NHANES website (https://wwwn.cdc.gov/Nchs/Nhanes/2001-2002/L06VIT_B. $\mathrm{htm})$ and in the National Report on Biochemical Indicators of Diet and Nutrition in the US Population 1999-2002. ${ }^{12}$

\section{Covariates}

The demographic data of our study population included age, race, health and medical information (smoking, congestive heart failure, coronary heart disease or episodes of angina/ angina pectoris heart disease, and cancer or malignancy). Biochemical parameters for creatinine $(\mathrm{Cr})$, alanine aminotransferase (ALT), total cholesterol, and C-reactive protein (CRP) were analyzed. The laboratory assay methods and protocols are described in detail on the NHANES website 
(https://wwwn.cdc.gov/nchs/nhanes/search/datapage.aspx? Component=Laboratory\&CycleBeginYear=2001).

\section{Statistical Analysis}

Our study used SPSS version 22 (SPSS Inc., Chicago, IL, USA) for statistical analysis. Nominal and quantitative variables are expressed as counts and percentages and as means \pm standard deviation (SD). The $\chi^{2}$ test was used to verify the relationship between serum nutritional biomarkers and HE4. Multivariable linear regression was used to compare changes in the regression coefficients of variables. Serum nutritional biomarker levels were divided into four equal parts, and the lowest quartile was set as the reference group. Analysis of variance (ANOVA) was used to measure the differences between continuous variables and HE4. A p value $<0.05$ was set as the cutoff that indicated statistical significance.

Three models were investigated to adjust relevant covariates. Model 1 was based on age and race; Model 2 was a modified version of Model 1 plus Cr, ALT, and CRP; and Model 3 was a modified version of Model 2 plus histories of smoking, heart disease, and cancer.

\section{Results}

There were 2464 eligible participants in our study. We excluded missing data of serum nutritional biomarkers and HE4. Serum nutritional biomarkers which statistical significance $\mathrm{p}$ value $<0.05$ was included and was divided into quartile in the analysis of variance (ANOVA).

Figure 1 shows a flow chart of our research. The characteristics of our study population were categorized based on the continuous variables and categorical variables in Table 1. Our participants were all female and had a mean age of 48.07 years old $(\mathrm{SD}=19.26)$. Creatinine, alanine aminotransferase and C-reactive protein were within normal ranges in our study participants. The participants had a slightly higher mean total cholesterol level $(204.98 \pm 42.24 \mathrm{mg} / \mathrm{dL})$. NonHispanic whites comprised $52.8 \%$, which was more than non-Hispanic black and other races.

Table 2 and Figure 2 show the associations between serum nutritional biomarkers and human epididymis protein 4. After additional adjustment in Model 3 (age + race + Cholesterol $+\mathrm{Cr}+\mathrm{ALT}+\mathrm{CRP}+$ History of smoking + History of heart disease + History of cancer), the serum nutritional biomarkers that were statistically significant were $\alpha$-carotene, trans- $\beta$-carotene, cis- $\beta$ carotene, trans-lycopene and retinol. Their beta coefficients were $-0.102,-0.027,-0.506,-0.131$ and -0.054 , respectively.

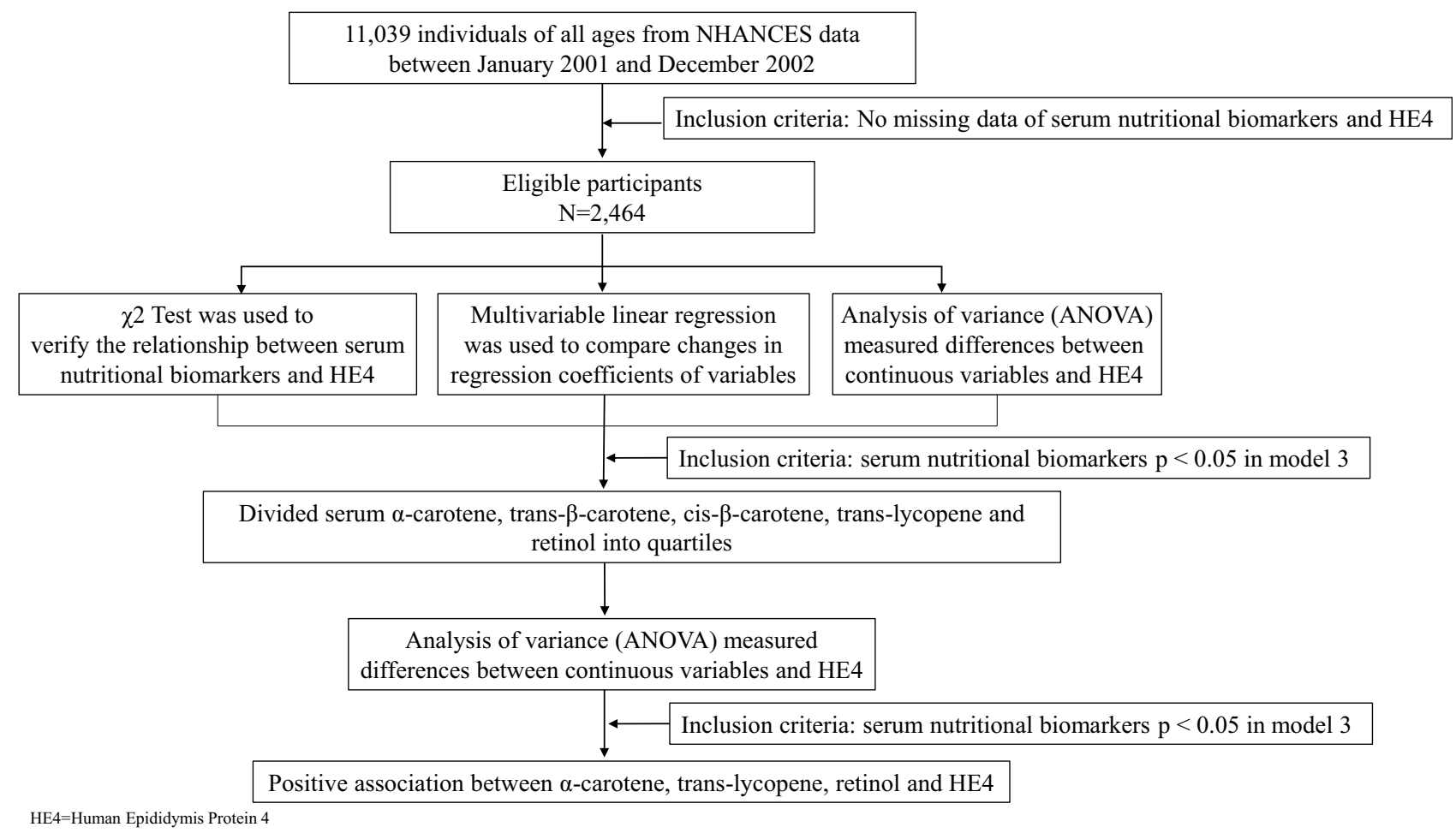

Figure I Flow chart. 
Table I Characteristics of Study Participants

\begin{tabular}{|l|l|}
\hline Characteristic & Total Population N=2464 \\
\hline Continuous variables* & \\
Age (years) & $48.07(19.26)$ \\
Creatinine (mg/dL) & $0.78(0.43)$ \\
ALT(U/L) & $21.19(40.02)$ \\
Total cholesterol (mg/dL) & $204.98(42.24)$ \\
CRP (mg/dL) & $0.57(0.96)$ \\
Vitamin D (ng/mL) & $21.65(9.76)$ \\
$\alpha$-carotene (ug/dL) & $4.73(6.95)$ \\
Trans- $\beta$-carotene (ug/dL) & $20.97(21.48)$ \\
Cis- $\beta$-carotene (ug/dL) & $1.23(1.23)$ \\
$\beta$-cryptoxanthin (ug/dL) & $11.05(9.71)$ \\
$\gamma$-tocopherol (ug/dL) & $232.60(133.25)$ \\
Lutein/zeaxanthin (ug/dL) & $16.52(9.16)$ \\
Trans-lycopene (ug/dL) & $21.58(10.50)$ \\
Retinyl palmitate (ug/dL) & $2.44(2.7 I)$ \\
Retinyl stearate (ug/dL) & $0.49(0.87)$ \\
Retinol (ug/dL) & $56.14(18.25)$ \\
$\alpha$-tocopherol (ug/dL) & $1378.10(653.74)$ \\
\hline Categorical variables ${ }^{\dagger}$ & \\
Non-Hispanic White & $1302(52.8)$ \\
\hline Smoking & \\
Congestive heart failure & $60(2.4)$ \\
Coronary heart disease & $68(2.8)$ \\
Angina/Angina pectoris & $79(3.2)$ \\
Cancer or malignancy & $214(8.7)$ \\
\hline
\end{tabular}

Notes: I missing data; *Continuous variables are presented as mean (standard deviation); ${ }^{\dagger}$ Categorical variables are presented as number (percentage). Abbreviations: CRP, C-reactive protein; ALT, alanine aminotransferase.
A Dose-Dependent Association Exists Between $\alpha$-Carotene, Trans-Lycopene, Retinol, and Human Epididymis Protein 4 Table 3 shows the association between the quartiles of serum nutritional biomarkers and human epididymis protein 4 . A quartile-based analysis was performed by dividing the serum nutritional biomarkers into quartiles. The first quartile was set as our reference group. A negative correlation between $\alpha$-carotene, trans-lycopene and retinol was observed after additional adjustment in the three models. The trends were statistically significant in the three models. The beta coefficients of Q4 versus Q1 for $\alpha$-carotene were -1.965 in Model 1 (age + race),-1.795 in Model 2 (Model $1+$ Cholesterol $+\mathrm{Cr}+$ ALT + CRP) and -3.390 in Model 3 (Model $2+$ History of smoking + History of heart disease + History of cancer). The beta coefficients of Q4 versus Q1 for trans-lycopene were -3.948 in Model 1, -4.328 in Model 2 and -4.036 in Model 3. The beta coefficients of retinol between Q4 and Q1 were -2.867 in Model 1, -4.146 in Model 2 and -4.146 in Model 3. The models had similar $\mathrm{p}$ trends of $<0.001$.

\section{Discussion}

Our results demonstrated a negative association between micronutrients and serum HE4 concentration in healthy subjects. Decreases in the concentration of HE4 were accompanied by significant increases in $\alpha$-carotene, trans-lycopene

Table 2 Associations Between Serum Nutritional Biomarkers and Human Epididymis Protein 4

\begin{tabular}{|c|c|c|c|c|c|c|}
\hline Variables & $\begin{array}{c}\text { Model I } \\
\beta^{b}(95 \% \text { CI })\end{array}$ & P-value & $\begin{array}{c}\text { Model } 2^{a} \\
\beta^{b}(95 \% \text { Cl })\end{array}$ & P-value & $\begin{array}{c}\text { Model } 3 \\
\beta^{b}(95 \% \mathrm{Cl})\end{array}$ & P-value \\
\hline Vitamin D & $0.060(-0.028,0.149)$ & 0.178 & $0.023(-0.034,0.08 \mathrm{I})$ & 0.423 & $0.017(-0.040,0.074)$ & 0.561 \\
\hline$\alpha$-Carotene & $-0.236(-0.36 \mathrm{I},-0.1 \mathrm{II})$ & $<0.001$ & $-0.125(-0.207,-0.042)$ & 0.003 & $-0.102(-0.184,-0.020)$ & 0.014 \\
\hline Trans- $\beta$-carotene & $-0.067(-0.108,-0.026)$ & 0.001 & $-0.035(-0.062,-0.008)$ & 0.012 & $-0.027(-0.054,0.000)$ & 0.048 \\
\hline Cis- $\beta$-carotene & $-1.167(-1.886,-0.449)$ & 0.001 & $-0.635(-1.111,-0.159)$ & 0.009 & $-0.506(-0.980,-0.033)$ & 0.036 \\
\hline$\beta$-cryptoxanthin & $-0.142(-0.234,-0.05 \mathrm{I})$ & 0.002 & $-0.04 \mathrm{I}(-0.10 \mathrm{I}, 0.020)$ & 0.192 & $-0.004(-0.065,0.058)$ & 0.909 \\
\hline$\gamma$-tocopherol & $0.009(0.003,0.015)$ & 0.006 & $0.001(-0.004,0.005)$ & 0.696 & $0.000(-0.004,0.005)$ & 0.905 \\
\hline Lutein/zeaxanthin & $-0.182(-0.277,-0.087)$ & $<0.001$ & $-0.050(-0.116,0.016)$ & 0.137 & $-0.023(-0.089,0.043)$ & 0.493 \\
\hline Trans-lycopene & $-0.185(-0.269,-0.101)$ & 0.000 & $-0.131(-0.190,-0.072)$ & $<0.001$ & $-0.131(-0.190,-0.073)$ & 0.000 \\
\hline Retinyl palmitate & $0.140(-0.467,0.187)$ & 0.402 & $-0.087(-0.302,0.128)$ & 0.429 & $-0.063(-0.277,0.150)$ & 0.560 \\
\hline Retinyl stearate & $-0.055(-1.067,0.957)$ & 0.915 & $-0.05 \mathrm{I}(-0.707,0.606)$ & 0.880 & $-0.019(-0.669,0.631)$ & 0.955 \\
\hline Retinol & $0.297(0.247,0.347)$ & $<0.001$ & $-0.043(-0.079,-0.007)$ & 0.020 & $-0.054(-0.090,-0.018)$ & 0.004 \\
\hline$\alpha$-tocopherol & $0.002(0.000,0.003)$ & 0.013 & $0.000(-0.001,0.001)$ & 0.593 & $0.00 \mathrm{I}(-0.00 \mathrm{I}, 0.002)$ & 0.347 \\
\hline
\end{tabular}

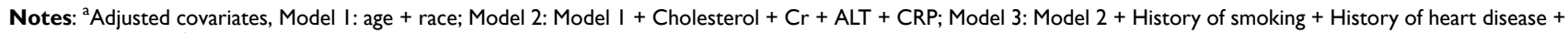
History of cancer; ${ }^{b} \beta$ coefficients was interpreted as change of log-transformed human epididymal secretory protein ( $p M$ ) for each increase in different serum nutritional biomarkers.

Abbreviations: CRP, C-reactive protein; ALT, alanine aminotransferase. 


\begin{tabular}{|c|c|}
\hline Vitamin D & $0.060(-0.028,0.149)$ \\
\hline$\alpha$-carotene & $-0.236(-0.361,-0.111)$ \\
\hline trans- $\beta$-carotene & $-0.067(-0.108,-0.026)$ \\
\hline cis- $\beta$-carotene & $-1.167(-1.886,-0.449)$ \\
\hline$\beta$-cryptoxanthin & $-0.142(-0.234,-0.051)$ \\
\hline$\gamma$-tocopherol & $0.009(0.003,0.015)$ \\
\hline Lutein/zeaxanthin & $-0.182(-0.277,-0.087)$ \\
\hline trans-lycopene & $-0.185(-0.269,-0.101)$ \\
\hline Retinyl palmitate & $0.140(-0.467,0.187)$ \\
\hline Retinyl stearate & $-0.055(-1.067,0.957)$ \\
\hline Retinol & $0.297(0.247,0.347)$ \\
\hline$\alpha$-tocopherol & $0.002(0.000,0.003)$ \\
\hline Vitamin D & $0.023(-0.034,0.081)$ \\
\hline$\alpha$-carotene & $-0.125(-0.207,-0.042)$ \\
\hline trans- $\beta$-carotene & $-0.035(-0.062,-0.008)$ \\
\hline cis- $\beta$-carotene & $-0.635(-1.111,-0.159)$ \\
\hline$\beta$-cryptoxanthin & $-0.041(-0.101,0.020)$ \\
\hline$\gamma$-tocopherol & $0.001(-0.004,0.005)$ \\
\hline Lutein/zeaxanthin & $-0.050(-0.116,0.016)$ \\
\hline trans-lycopene & $-0.131(-0.190,-0.072)$ \\
\hline Retinyl palmitate & $-0.087(-0.302,0.128)$ \\
\hline Retinyl stearate & $-0.051(-0.707,0.606)$ \\
\hline Retinol & $-0.043(-0.079,-0.007)$ \\
\hline$\alpha$-tocopherol & $0.000(-0.001,0.001)$ \\
\hline Vitamin D & $0.017(-0.040,0.074)$ \\
\hline$\alpha$-carotene & $-0.102(-0.184,-0.020)$ \\
\hline trans- $\beta$-carotene & $-0.027(-0.054,0.000)$ \\
\hline cis- $\beta$-carotene & $-0.506(-0.980,-0.033)$ \\
\hline$\beta$-cryptoxanthin & $-0.004(-0.065,0.058)$ \\
\hline$\gamma$-tocopherol & $0.000(-0.004,0.005)$ \\
\hline Lutein/zeaxanthin & $-0.023(-0.089,0.043)$ \\
\hline trans-lycopene & $-0.131(-0.190,-0.073)$ \\
\hline Retinyl palmitate & $-0.063(-0.277,0.150)$ \\
\hline Retinyl stearate & $-0.019(-0.669,0.631)$ \\
\hline Retinol & $-0.054(-0.090,-0.018)$ \\
\hline$\alpha$-tocopherol & $0.001(-0.001,0.002)$ \\
\hline
\end{tabular}

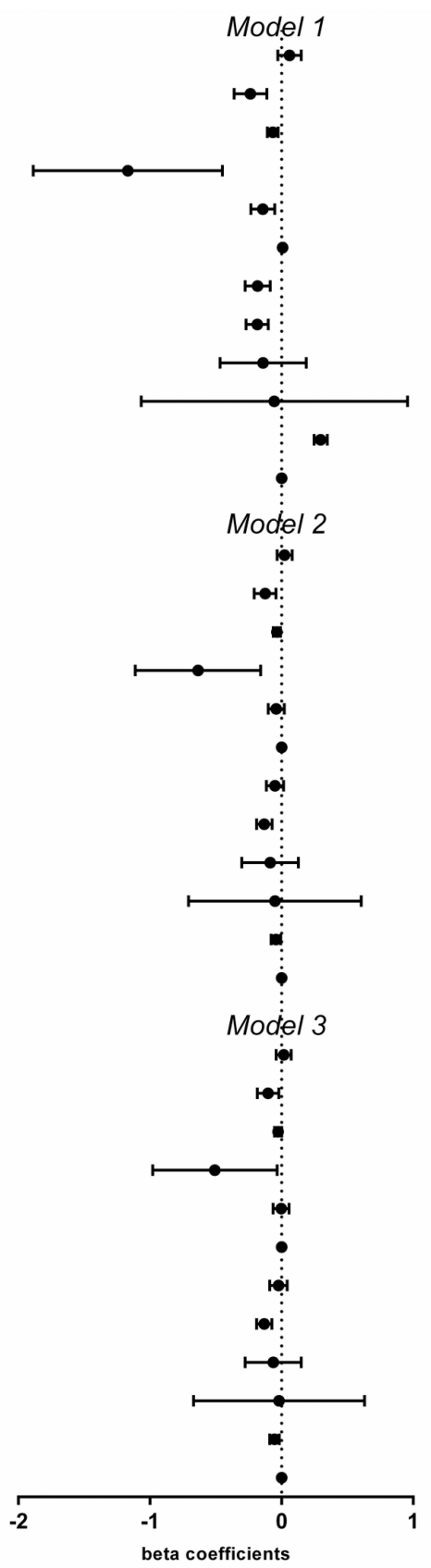

Figure 2 Associations between serum nutritional biomarkers and human epididymis protein 4 .

and retinol. Although adjustment for confounders in Model 3 reduced the magnitude of the effects, there were nonlinear increasing trends for these micronutrients with reductions in HE4 levels ( $p$ trend $<0.01$ ). These findings support micronutrients in the diet because a healthier lifestyle influenced the serum HE4 concentration. Focusing on gaps in knowledge, in this study, we were interested in the molecular signaling of $\alpha$-carotene, trans-lycopene, retinol, and HE4.

HE4 was first recognized in the distal epididymis. ${ }^{13}$ It was also found in the glandular epithelium of the salivary glands, trachea, lung and nasal epithelium, distal renal tubules, vas deferens, and female genital tract. ${ }^{14}$ HE4, which is coexpressed with antileukoproteinase and peptidase inhibitor 3, may further take part in the innate immunity of the oro-, nasopharynx and respiratory tract. ${ }^{15}$ Moreover, due to its significant correlation with interleukin-6 (IL-6), HE4 was used as an indicator of the onset of smell and/or taste disorder and the evaluation of multiorgan involvement in the clinical course of COVID-19. ${ }^{16}$ In addition, HE4 is also a protease inhibitor that degrades 


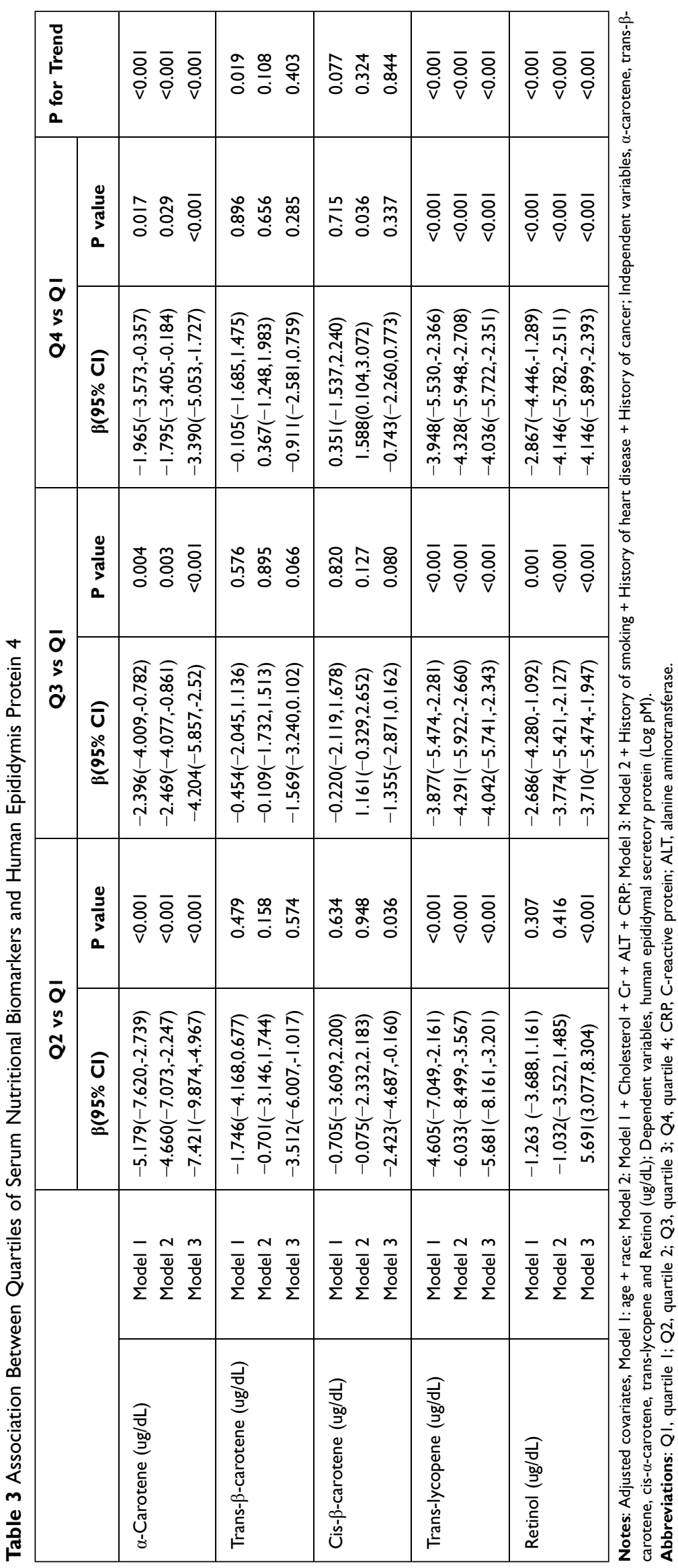


type I collagen to prevent kidney fibrosis by decreasing serine protease activity. ${ }^{17}$

HE4 is capable of upregulating the expression of genes involved in anabolic and catabolic pathways in epithelial ovarian cancer cell lines, especially SKOV3 and OVCAR8 cells via the MAPK/ERK, PI3K/AKT and hypoxiainducible factor (HIF) $1 \alpha$ pathways. ${ }^{7}$ Moreover, it can suppress estrogen signaling in steroid biosynthesis. ${ }^{7}$ The mechanism includes epigenetic modification and nuclear importation. ${ }^{4}$ The differential expression of several genes involved in growth/proliferation, metastasis, chemoresistance, and steroid biosynthesis suggests that HE4 may ultimately contribute to tumorigenesis. ${ }^{7}$

Although making serum HE4 a new biomarker a reality is feasible, there are some factors that influence the accuracy of the HE4 concentration. The factors are as follows: parity, caffeine consumption, use of oral contraceptives, older age, smoking, and the impairment of renal function. ${ }^{18}$ Before using HE4 in clinical applications, there was still some information that needed to be determined. ${ }^{19}$ One question was to identify the lifestyle and biological factors that influence serum HE4 concentration. ${ }^{19}$ Our study found independent inverse associations between serum $\alpha$-carotene, trans-lycopene, retinol and HE4.

\section{Properties and Biological Activities of Microalgal Carotenoids}

Most carotenoids are obtained from plants, vegetables, and fruits or are synthesized by microalgae. Most microalgae protect themselves from oxidative injury by transferring excess light energy and scavenging reactive oxygen species (ROS) to nearby chlorophyll-protein complexes. ${ }^{20}$ Although carotenoids are mostly extracted from microalgae that are involved in light-harvesting functions, their different and unique structures give them different properties. ${ }^{20}$ Scientists have reviewed their various antagonistic biological activities, such as angiogenesis and inflammatory and oxidizing properties. ${ }^{20}$

Haematococcus pluvialis accumulates the orange-red pigment astaxanthin as the main carotenoid, as well as other related carotenoids, including $\beta$-carotene and lutein. ${ }^{20}$ Carotenoids produced by $\mathrm{H}$. pluvialis are widely used in the prevention and treatment of benign prostatic hyperplasia and prostate and liver tumors. ${ }^{20}$ However, in our results, $\beta$-carotene and lutein did not show a significant effect on HE4 concentration. This may be due to the lower expression of HE4 in the prostate and liver. ${ }^{21}$
Dunaliella salina is well known for being rich in $\beta$ carotene, lutein, zeaxanthin and $\alpha$-carotene. The beneficial effects of these carotenes include the prevention of colorectal cancer, acute and chronic coronary syndrome, and liver fibrosis. The micronutrients contained in D. salina also act as provitamin A carotenoids that are converted into vitamin A. ${ }^{20}$ Therefore, they might share a similar effect on the HE4 concentration. In our results, lutein/zeaxanthin showed a significant $p$ value in adjusted Model 1 (Table 2), while $\beta$ carotene showed a significant $\mathrm{p}$ value in adjusted Models 1, 2 and 3 (Table 2), although this effect was not apparent in the quartile-based analysis. In contrast, $\alpha$-carotene had a significant effect on the HE4 concentration in the quartilebased analysis. Clinical trials have shown that isolating $\alpha$ carotene is difficult given the ample amounts of $\beta$-carotene in fruits and vegetables. ${ }^{22}$ Therefore, our results have determined the value of $\alpha$-carotene on the effect of HE4 concentration independent from $\beta$-carotene.

$\beta$-Carotene is the most studied provitamin A carotenoid in terms of its metabolic pathway and potential effects on health. Therefore, we chose $\beta$-carotene as the representative carotenoid. $\beta$-Carotene has antiangiogenic effects that can inhibit the formation of tumor-directed capillaries by altering serum cytokine levels. ${ }^{23}$ However, its anticarcinogenic activity was found to be lower than those of $\alpha$-carotene, $\beta$ cryptoxanthin, lutein, zeaxanthin, and lycopene. ${ }^{24}$ Therefore, $\alpha$-carotene and lycopene may have greater contributions than $\beta$-carotene to the HE4 concentration.

In addition, scientists have demonstrated that high serum concentrations of carotenoids obtained from food are associated with a decreased risk of chronic disease. ${ }^{25}$ However, these benefits sometimes disappear when extracted from the plant matrix. ${ }^{22}$ For example, scientists have conducted two clinical trials and shown an increased lung cancer risk with high-dose $\beta$-carotene from food sources due to the increased formation of abnormal tissue in the lung. ${ }^{26,27}$ However, another study demonstrated that the median concentrations of $\beta$-carotene, lycopene and lutein reduced the risk of breast cancer. ${ }^{22}$ As a result, the effect of micronutrients on cancer might be concentrationand organ-dependent.

\section{The Possible Mechanisms Associated with $\alpha$-Carotene, Trans-Lycopene, Retinol and HE4}

$\alpha$-Carotene is a type of carotenoid and an important precursor of vitamin $\mathrm{A}^{28}$ It is a fat-soluble carotenoid 
pigment that is mostly found in yellow-orange and darkgreen vegetables. ${ }^{29}$ In contrast to $\beta$-carotene, $\alpha$-carotene is an aliphatic hydrocarbon that contains four terpene subunits with a single retinyl group. ${ }^{28}$ It has antioxidant, anticarcinogenic, and cardiovascular protective properties. ${ }^{28} \alpha$-Carotene more efficiently inhibits invasion, migration, and adhesion, as well as the attenuation of the ERK, JNK and p38-Mapk pathways in human hepatocarcinoma than does $\beta$-carotene. ${ }^{30}$

Trans-lycopene has greater anticancer ability than do other carotenoids. $^{24}$ Although it lacks provitamin A activity, it has greater antioxidant potential than $\beta$ carotene and $\alpha$-tocopherol. ${ }^{31}$ It is widely distributed in the body, including in the testes, liver, adrenal glands, and kidneys. ${ }^{22}$ In addition, it is associated with a reduced risk of prostate, bladder, cervical, gastrointestinal tract and breast cancers. ${ }^{22}$ In addition, it neutralizes free radicals before they destroy the collagen and elastin fibers in the skin. Therefore, it is also used as a skin supplement. ${ }^{32}$

In addition, both $\alpha$-carotene and lycopene act as antioxidants and blue light filters and can relieve sleep-related symptoms. ${ }^{33}$ Lycopene suppresses the proliferation of SKOV3 ovarian cancer cells and accelerates and induces apoptosis by regulating the expression of Bax and Bcl-2. ${ }^{34}$ In addition, lycopene plays a role in delaying the S-phase transition and reducing $\mathrm{G} 0 / \mathrm{G} 1$ phase in the cell cycle in ultraviolet-B-irradiated human keratinocytes. ${ }^{35}$ Scientists have demonstrated that lycopene improves heart function and ventricular remodeling by inhibiting myocardialinfarction-induced increases in MMP-9 and p38 activation and attenuating the nuclear factor-кb signaling pathway in the inflammatory response and in cardiomyocyte apoptosis. ${ }^{36}$

Retinol is the most common molecule converted to vita$\min \mathrm{A}$ and is enriched in the fat component of dairy products. ${ }^{37}$ It contributes to the differentiation of epithelial tissue and bone, as well as reproduction and the immune response. ${ }^{37}$ It also plays an important role in the gene transcription of the retinoic acid receptor (RAR). ${ }^{38}$ Scientists have demonstrated that the RAR gene is involved in nonrapid eye movement sleep in mouse sleep electroencephalograms (EEGs). ${ }^{39}$ Vitamin A and RAR antagonists can reverse shortened sleep-wake cycles. ${ }^{40} \alpha$-Carotene, trans-lycopene, and retinol, which showed similar effects on sleep-related symptoms, might share similar effects on the concentration of HE4.

All-trans retinoic acid (ATRA), a vitamin A derivative, is the most important drug for treating acute promyelocytic leukemia by targeting PML/RAR $\alpha$ abnormalities. ${ }^{41}$ It has therapeutic potential in several cancers: in colorectal cancer, it can inhibit the activation of the MAPK/ERK signaling pathway; in melanoma, it can increase the expression of cyclin D1 mRNA; and in neuroblastoma, it can act via the PI3K and ERK1/2 pathways. ${ }^{42,43}$ In addition, ATRA promotes apoptosis, induces autophagy, and reverses the epithelial-mesenchymal transition by regulating Bcl-2 and its phosphorylated form in hepatocarcinoma cells. ${ }^{42}$

\section{Limitations}

Our study has several limitations. First, the major storage site of carotenoids is adipose tissue; therefore, adipose tissue would be a better predictor than would be concentrations in a blood analysis. ${ }^{44}$ However, we could only obtain blood profiles from the NHANES data. Second, we did not adjust the micronutrient levels based on body mass index (BMI) and alcohol levels. ${ }^{45}$ This is because both high alcohol levels and BMI were not significant confounders in our models. Third, we cannot determine the exact mechanism connecting $\alpha$-carotene, trans-lycopene, retinol, and HE4. However, because both micronutrients and HE4 have an important role in the cancer field, these micronutrients may directly or indirectly impact HE4 via cell proliferation, metastasis, chemoresistance and steroid biosynthetic pathways. ${ }^{7}$

\section{Conclusion}

$\alpha$-Carotene, trans-lycopene, and retinol were dosedependent and negatively associated with HE4 concentration. Supplement of micronutrients may reduce the levels of HE4 and the subsequent of ovarian cancer's risk. Although HE4 plays a key role in ovarian cancer, its therapeutic target potential is still underinvestigated. Therefore, to generate a formula correlating HE4 with micronutrients for clinical practice, studies are needed in a variety of populations, including patients with higher parity, caffeine consumption, use of oral contraceptives, older age, heavy smoking, and impaired renal function.

\section{Abbreviations}

NHANES, National Health and Nutrition Examination Survey; HE4, human epididymis protein 4; CA-125, cancer antigen 125; MAPK/ERK, mitogen activated protein kinase/ extracellular-signal-regulated kinase; MEC, mobile examination center; IRB, Institutional Review Board; NCHS, National Center for Health Statistics; Cr, creatinine; ALT, alanine aminotransferase; CRP, C-reactive protein; SD, standard deviation; ANOVA, analysis of variance; IL-6, 
interleukin-6; COVID-19, coronavirus disease 2019; PI3K AKT, phosphatidylinositol-3-kinase/protein kinase B; HIF, hypoxia-inducible factors; ROS, reactive oxygen species; MMP-9, matrix metallopeptidase 9; RAR, retinoic acid receptor; ATRA, All-trans retinoic acid; EEG, electroencephalogram.

\section{Data Sharing Statement}

All files are available from the NHANES database, https:// www.cdc.gov/nchs/nhanes/index.htm.

\section{Ethical Approval}

The NHANES study protocol was approved by the National Center for Health Statistics (NCHS) Institutional Review Board (IRB).

\section{Consent to Participate and to Publish Data}

Informed consent was obtained from all individual participants.

\section{Disclosure}

The authors have declared that no competing interests exist.

\section{References}

1. Barani M, Bilal M, Sabir F, Rahdar A, Kyzas GZ. Nanotechnology in ovarian cancer: diagnosis and treatment. Life Sci. 2020;266:118914.

2. Funston G, Hamilton W, Abel G, Crosbie EJ, Rous B, Walter FM. The diagnostic performance of CA125 for the detection of ovarian and non-ovarian cancer in primary care: a population-based cohort study. PLoS Med. 2020;17:e1003295. doi:10.1371/journal.pmed.1003295

3. Nolen BM, Lokshin AE. Protein biomarkers of ovarian cancer: the forest and the trees. Future Oncol. 2012;8:55-71. doi:10.2217/ fon. 11.135

4. Hertlein L, Stieber P, Kirschenhofer A, et al. Human epididymis protein 4 (HE4) in benign and malignant diseases. Clin Chem Lab Med. 2012;50:2181-2188. doi:10.1515/cclm-2012-0097

5. Saini RK, Keum YS, Daglia M, Rengasamy KR. Dietary carotenoids in cancer chemoprevention and chemotherapy: a review of emerging evidence. Pharmacol Res. 2020;157:104830. doi:10.1016/j.phrs.2020.1 04830

6. Beydoun MA, Gamaldo AA, Canas JA, et al. Serum nutritional biomarkers and their associations with sleep among US adults in recent national surveys. PLoS One. 2014;9:e103490. doi:10.1371/journal.pone.0103490

7. James NE, Chichester C, Ribeiro JR. Beyond the biomarker: understanding the diverse roles of human epididymis protein 4 in the pathogenesis of epithelial ovarian cancer. Front Oncol. 2018;8:124. doi: $10.3389 /$ fonc. 2018.00124

8. Centers for Disease Control and Prevention (CDC). National Center for Health Statistics (NCHS). National health and nutrition examination survey data. Hyattsville, MD: U.S. Department of Health and Human Services, Centers for Disease Control and Prevention, [20012002]; Available from: https://www.cdc.gov/nchs/nhanes/index.htm. Accessed September 21, 2021.
9. Ballew C, Bowman BA, Sowell AL, Gillespie C. Serum retinol distributions in residents of the United States: third National Health and Nutrition Examination Survey, 1988-1994. Am J Clin Nutr. 2001;73:586-593. doi:10.1093/ajcn/73.3.586

10. Ford ES, Gillespie C, Ballew C, Sowell A, Mannino DM. Serum carotenoid concentrations in US children and adolescents. Am J Clin Nutr. 2002;76:818-827. doi:10.1093/ajen/76.4.818

11. Pfeiffer CM, Sternberg MR, Schleicher RL, Haynes BM, Rybak ME, Pirkle JL. The CDC's second national report on biochemical indicators of diet and nutrition in the US population is a valuable tool for researchers and policy makers. $J$ Nutr. 2013;143:938S-947S. doi: $10.3945 /$ jn. 112.172858

12. Pfeiffer CM, Schleicher RL, Osterloh JD, Jain RB, Wong LY, Sampson EJ. National report on biochemical indicators of diet and nutrition in the US population 1999-2002. FASEB J. 2009;23:551.26. doi:10.1096/fasebj.23.1_supplement.551.26

13. Bingle L, Singleton V, Bingle CD. The putative ovarian tumour marker gene HE4 (WFDC2), is expressed in normal tissues and undergoes complex alternative splicing to yield multiple protein isoforms. Oncogene. 2002;21:2768-2773. doi:10.1038/sj.onc. 1205363

14. Galgano MT, Hampton GM, Frierson HF. Comprehensive analysis of HE4 expression in normal and malignant human tissues. Mod Pathol. 2006;19:847-853. doi:10.1038/modpathol.3800612

15. Bingle L, Cross SS, High AS, et al. WFDC2 (HE4): a potential role in the innate immunity of the oral cavity and respiratory tract and the development of adenocarcinomas of the lung. Respir Res. 2006;7:1-10. doi:10.1186/1465-9921-7-61

16. Schirinzi A, Cazzolla AP, Lovero R, et al. New insights in laboratory testing for COVID-19 patients: looking for the role and predictive value of Human epididymis secretory protein 4 (HE4) and the innate immunity of the oral cavity and respiratory tract. Microorganisms. 2020;8:1718. doi:10.3390/microorganisms8111718

17. LeBleu VS, Teng Y, T O'Connell J, et al. Identification of human epididymis protein-4 as a fibroblast-derived mediator of fibrosis. Nat Med. 2013;19:227-231. doi:10.1038/nm.2989

18. Ferraro S, Pasqualetti S, Carnevale A, Panteghini M. Cystatin C provides a better estimate of the effect of glomerular filtration rate on serum human epididymis protein 4 concentrations. Clin Chem Lab Med. 2016;54:1629-1634. doi:10.1515/cclm-2015-1272

19. Ferraro $S$, Panteghini M. Is serum human epididymis protein 4 ready for prime time? Ann Clin Biochem. 2014;51:128-136. doi:10.1177/ 0004563213500657

20. Sathasivam R, Ki JS. A review of the biological activities of microalgal carotenoids and their potential use in healthcare and cosmetic industries. Mar Drugs. 2018;16:26. doi:10.3390/md16010026

21. Simmons AR, Baggerly K, Bast RC. The emerging role of HE4 in the evaluation of advanced epithelial ovarian and endometrial carcinomas. Oncology. 2013;27:548.

22. Burri B. Carotenoids: chemistry, sources and physiology. Encycl Hum Nutr. 2013;1:283-291.

23. Guruvayoorappan C, Kuttan G. $\beta$-carotene inhibits tumor-specific angiogenesis by altering the cytokine profile and inhibits the nuclear translocation of transcription factors in B16F-10 melanoma cells. Integr Cancer Ther. 2007;6:258-270. doi:10.1177/1534735407 305978

24. Nishino H, Murakoshi M, Ii T, et al. Carotenoids in cancer chemoprevention. Cancer Metastasis Rev. 2002;21:257-264. doi:10.1023/A:1021206826750

25. Raposo MF, De Morais AM, De Morais RM. Carotenoids from marine microalgae: a valuable natural source for the prevention of chronic diseases. Mar Drugs. 2015;13:5128-5155. doi:10.3390/ md 13085128

26. Goodman GE, Omenn GS, Coinvestigators C. Carotene and retinol efficacy trial: lung cancer chemoprevention trial in heavy cigarette smokers and asbestos-exposed workers. In: The Biology and Prevention of Aerodigestive Tract Cancers. Springer; 1992:137-140. 
27. Albanes D, Heinonen OP, Huttunen JK, et al. Effects of alpha-tocopherol and beta-carotene supplements on cancer incidence in the Alpha-Tocopherol Beta-Carotene Cancer Prevention Study. Am J Clin Nutr. 1995;62:1427S-1430S. doi:10.1093/ajcn/62.6.1427S

28. Tanumihardjo S Carotenoids: health effects. 2013.

29. Voutilainen S, Nurmi T, Mursu J, Rissanen TH. Carotenoids and cardiovascular health. Am J Clin Nutr. 2006;83:1265-1271. doi:10.1093/ajen/83.6.1265

30. Chen HY, Yueh TC, Chen YC, Huang CH, Yang CM, Hu ML. Antimetastatic effects of $\alpha$-carotene and possible mechanisms of action in human hepatocarcinoma SK-Hep-1 cells. J Agric Food Chem. 2013;61:10368-10376. doi:10.1021/jf4033393

31. Tapiero H, Townsend DM, Tew KD. The role of carotenoids in the prevention of human pathologies. Biomed Pharmacother. 2004;58:100-110. doi:10.1016/j.biopha.2003.12.006

32. Hsieh HY, Lee WC, Senadi GC, et al. Discovery, synthetic methodology, and biological evaluation for antiphotoaging activity of bicyclic $[1,2,3]$ triazoles: in vitro and in vivo studies. $J \mathrm{Med}$ Chem. 2013;56:5422-5435. doi:10.1021/jm400394s

33. Grandner MA, Jackson N, Gerstner JR, Knutson KL. Sleep symptoms associated with intake of specific dietary nutrients. J Sleep Res. 2014;23:22-34. doi:10.1111/jsr.12084

34. Xu J, Li Y, Hu H. Effects of lycopene on ovarian cancer cell line SKOV3 in vitro: suppressed proliferation and enhanced apoptosis. Mol Cell Probes. 2019;46:101419. doi:10.1016/j.mcp.2019.07.002

35. Ascenso A, Pedrosa T, Pinho S, et al. The effect of lycopene preexposure on UV-B-irradiated human keratinocytes. Oxid Med Cell Longev. 2016;2016:1-15. doi:10.1155/2016/8214631

36. He Q, Zhou W, Xiong C, Tan G, Chen M. Lycopene attenuates inflammation and apoptosis in post-myocardial infarction remodeling by inhibiting the nuclear factor- $\kappa \mathrm{B}$ signaling pathway. Mol Med Rep. 2015;11:374-378. doi:10.3892/mmr.2014.2676
37. Blaner WS. Chapter 5 - vitamin A and provitamin A carotenoids. In: Marriott BP, Birt DF, Stallings VA, Yates AA, editors. Present Knowledge in Nutrition. 11th ed. Academic Press; 2020:73-91.

38. Mey J, Mccaffery P. Retinoic acid signaling in the nervous system of adult vertebrates. Neuroscientist. 2004;10:409-421. doi:10.1177/ 1073858404263520

39. Maret S, Franken P, Dauvilliers Y, Ghyselinck NB, Chambon P, Tafti M. Retinoic acid signaling affects cortical synchrony during sleep. Science. 2005;310:111-113. doi:10.1126/science.1117623

40. Kitaoka K, Shimizu M, Shimizu N, et al. Retinoic acid receptor antagonist LE540 attenuates wakefulness via the dopamine D1 receptor in mice. Brain Res. 2011;1423:10-16. doi:10.1016/j. brainres.2011.09.023

41. Zhou GB, Zhang J, Wang ZY, Chen SJ, Chen Z. Treatment of acute promyelocytic leukaemia with all-trans retinoic acid and arsenic trioxide: a paradigm of synergistic molecular targeting therapy. Philos Trans $R$ Soc Lond B Biol Sci. 2007;362:959-971. doi:10.1098/rstb.2007.2026

42. Jobani BM, Najafzadeh N, Mazani M, Arzanlou M, Vardin MM. Molecular mechanism and cytotoxicity of allicin and all-trans retinoic acid against CD44+ versus CD117+ melanoma cells. Phytomedicine. 2018;48:161-169. doi:10.1016/j.phymed.2018.05.013

43. Qiao J, Paul P, Lee S, et al. PI3K/AKT and ERK regulate retinoic acid-induced neuroblastoma cellular differentiation. Biochem Biophys Res Commun. 2012;424:421-426. doi:10.1016/j.bbrc.2012.06.125

44. Parker RS. Carotenoids in human blood and tissues. J Nutr. 1989;119:101-104. doi:10.1093/jn/119.1.101

45. Brady WE, Mares-Perlman JA, Bowen P, Stacewicz-Sapuntzakis M. Human serum carotenoid concentrations are related to physiologic and lifestyle factors. J Nutr. 1996;126:129-137. doi:10.1093/jn/ 126.1.129
Journal of Inflammation Research

\section{Publish your work in this journal}

The Journal of Inflammation Research is an international, peerreviewed open-access journal that welcomes laboratory and clinical findings on the molecular basis, cell biology and pharmacology of inflammation including original research, reviews, symposium reports, hypothesis formation and commentaries on: acute/chronic inflammation; mediators of inflammation; cellular processes; molecular

\section{Dovepress}

mechanisms; pharmacology and novel anti-inflammatory drugs; clinical conditions involving inflammation. The manuscript management system is completely online and includes a very quick and fair peerreview system. Visit http://www.dovepress.com/testimonials.php to read real quotes from published authors. 